\title{
APPLICATION OF INDUSTRIAL ENGINEERING THEORIES FOR A REAL MANUFACTURING PROCESS IMPROVEMENT USING SIMULATION MODELLING
}

\author{
Hernan D. Torres, Edwin A. Garavito Associated \\ Professors, Faculty of Industrial Engineering \\ Universidad Industrial de Santander, Cra. 27 No. 9 \\ Bucaramanga, Santander, Colombia \\ $(57-7)$ - 6344000
}

garavito@uis.edu.co,

hernan.torres@consultorresingenieria.com

\author{
Federico E. Rincon \\ Industrial Engineering Consultant \\ Consultorres Ingeniería S.A.S. Cra. 37 \# 52-43, Of. \\ 902, Bucaramanga, Santander, Colombia \\ $(57-7)$ - 6914514
}

federico.rincon@consultorresingenieria.com

\begin{abstract}
The purpose of this paper is to describe a detailed and sequential methodology in industrial processes improvement using simulation models. In order to strengthen this academic approach, its application is explained through a case study developed in a medium size company that manufactures windows, doors and facades established in Bucaramanga, Colombia. The article presents the description of the industrial process, the development of the simulation model and the experimental results obtained with its conclusions. The main benefits of this research include: First, the adjustment of basic industrial engineering terms of efficiency and capacity to the specialized simulation software ARENA $\AA$, second, the performance analysis of a complex manufacturing process, and finally, the techniques used for the improvement of the performance measures avoiding trial and error techniques.
\end{abstract}

\section{Categories and Subject Descriptors}

I.6.3. [Simulation and Modeling]: Applications

\section{General Terms}

Measurement, Performance, Experimentation, Verification.

\section{Keywords}

Simulation, Process Improvement, ARENA, Window and Facades Manufacturing.
Permission to make digital or hard copies of all or part of this work for personal or classroom use is granted without fee provided that copies are not made or distributed for profit or commercial advantage and that copies bear this notice and the full citation on the first page. To copy otherwise, to republish, to post on servers or to redistribute to lists, requires prior specific permission and/or a fee.

Simutools 2014, March 17-19, Lisbon, Portugal

Copyright $\odot 2014$ ICST 978-1-63190-007-5

DOI 10.4108/icst.simutools.2014.254738

\section{INTRODUCTION}

The computational simulation is described as models collection used through a defined methodology in order to represent a real o hypothetical process, being an excellent tool for the analysis of any manufacturing or service system [5]. Thus, simulation offers the best approach for understanding the systems dynamic, which is impossible by using static models, such as spreadsheets [3]. This technique offers the best cost/return rate in comparison to any other method applied with similar objectives [10]. Moreover, simulation is flexible enough for adapting "What If" scenarios, avoiding expensive trial and error procedures [1].

In this context, simulation clearly identifies and quantifies process flaws such as "bottlenecks" and productive wastes. A production waste is everything that does not meet the absolute minimum of equipment, materials, resources and labor work for producing a product or providing a service [17].

Therefore, the following paper describes the adaption of a simulation methodology to a real production process, obtaining remarkable results that strengthen the importance of using simulation for manufacturing process improvement.

\section{PROCESS IMPROVEMENT USING SIMULATION}

The flexibility provided in some software packages has allowed programmers to adapt simulation models to different areas, including: health systems, management, manufacturing, logistics and transportation [4], among others, making simulation a very versatile tool.

One of the biggest challenges for companies occurs during its planning process, where the future uncertainty plays an important role. The risks can be extremely high, considering that companies must invest large amounts of money in order to remain competitive. Therefore, engineering and science have developed techniques to predict future events based on statistics and historical data, generating mathematical models that reduce error chances. Hence, many companies have developed these models to the point of enhancing static models to probabilistic ones that consider the variability component associated with multiple factors depending on the type of the business [17]. 
Moreover, in order to strengthen the importance of simulation on processes improvement, few case studies were taken into account and are described as follows:

Osidach and $\mathrm{Fu}$ [15] describe the role of simulation in improving the efficiency of a Mobile Examination Center (MEC) in the United States that collects information about the national health. The simulation model was built in ARENA and its purpose was to assist the administrative operators on their planning decisions. With the simulation results; the process bottleneck was detected, the best configuration of resources was found and a better plant layout was proposed. The implementation of this project allowed increasing the total capacity and reducing the patients waiting time.

Furthermore, Mosca,Cassettari, Rivetria and Magro [14], described the application of a simulation model in ARENA in a medium-sized company which produces and assembles car components in Northern Italy. The main objective was to analyze in detail the system based on "What If" questions. The objective of the study was to determine the exact number of workers and machines needed. This analysis allowed managers to identify and implement strategies, achieving high performance in their production line.

Another example is based on a computer simulation model developed in Japan to analyze the behavior of fresh mixed concrete, accomplished by Tanigawa, Mori and Watanabe [18]. Using stochastic information, the model showed the analysts an idea of how the concrete behaves, considering different scenarios. The model is permanently reviewed and validated in real experiments with cylindrical rotation viscometers. Conclusions were based to contrast theoretical with practical values.

Therefore, the above mentioned and many other examples support the importance of the simulation applications, by demonstrating that with this methodology, notable results, such as: costs reduction, higher profits and better customer satisfaction, can be obtained.

\section{STUDY OVERVIEW}

\subsection{Production Plant}

This study was applied in a medium sized company named Ventanar S.A. that manufactures more than 130 references of aluminum windows, doors and facades and is located on the outskirts of Bucaramanga, Colombia. This company employs more than 200 workers and has a $90 \%$ of national coverage with an estimated capacity of $18.000 \mathrm{~m}^{2}$ of product per month. The layout of the production facility covers around $80,000 \mathrm{~m}^{2}$ and is distributed in the following main areas: Raw Materials Storage, Aluminum and Glass Cutting, Punch Pressing and Machining, Assembly and Packing.

\subsection{Manufacturing Process}

The manufacturing process in Ventanar S.A. is defined as a "Push" type system, although the demand is fully known. This results in high volumes of raw materials and in-process inventory. The production management programs the weekly orders and sets them on a specific day. The production orders are extremely personalized according to the construction project needs, turning this system in an accurate example of mass customization.

The process starts with the glass cutting. The glass is taken from the raw material storage, it is cut according to specifications and it is put on the in-process inventory. At the same time, the aluminum bar are cut and shaped with the measures required by the production order in a metal cutting machine; these profiles are then sent to the punch pressing and machining process. This last process consists on drilling the aluminum profiles with holes that allow supporting the whole structure and also facilitate the insertion of the opening and closing accessories. After all the above processes are finished, the cut glass and the drilled aluminum are manually assembled, packed and delivered for installation. Figure 1 shows the plant layout where the above described process takes place.

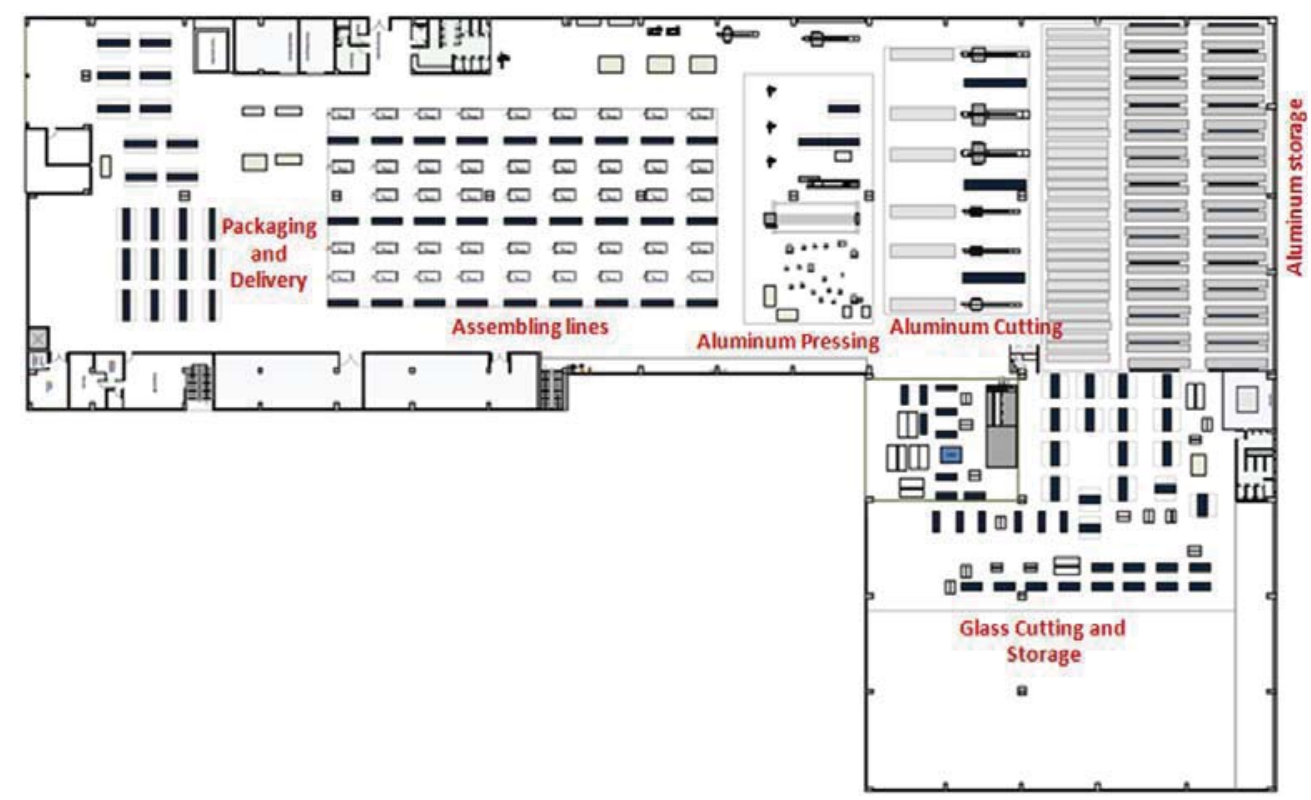

Figure 1. Ventanar S.A. Layout 


\section{SIMULATION MODEL}

\subsection{Assumptions}

Before constructing the model some assumptions were taken into account, in order to balance the real system excessive complexity and the simulation model final usefulness. This analysis followed the indications described by Harrell, Ghosh and Bowden [8]. Some of the relevant assumptions are highlighted below:

- The model is designed for representing normal conditions, eliminating atypical events like production peaks or major system failures. No extra working hours, sporadic absences or machines failures are considered. This assumption was taken to abolishing extraordinary events that impact the results in a negative way, as suggested by Garavito [6].

- The simulation runs for 172,800 minutes, representing a year of production (one shift of 600 minutes, 24 days per month).

- The simulation time was counted under a ten-hour shift per day, with continuous operation and a waste (supplement) of $10 \%$ as a result of non-productive work.

- A full provision of raw materials was assumed. No restrictions due to suppliers or other external agents were considered.

- Processing times and quantities distributions were obtained by collecting statistical data provided by Ventanar S.A. A total of 925 probability distributions were included as the result of a process of information compilation and analysis.

- A total of 16 groups of product references were taken into account. This allowed the possibility to recreate 52,250 different customized references at any time of the simulation.

- The assembling lines distribution was associated to different references, as shown in Table 1 . The model does not provide modulation between them, which means that there is no change of references per line in the case of slack or damming.

Table 11. Assembling line per reference

\begin{tabular}{|c|c|}
\hline ASSEMBLING LINE & TYPE OF SYSTEM \\
\hline 1 & Facades \\
\hline \multirow{4}{*}{2} & Tubular Frame Facades \\
\cline { 2 - 2 } & Pivot Doors \\
\cline { 2 - 2 } & Large Doors \\
\cline { 2 - 2 } & Other Products \\
\hline \multirow{4}{*}{3} & Maximum System Door \\
\cline { 2 - 2 } & Ibiza System Door \\
\cline { 2 - 2 } & Ibiza System Window \\
\cline { 2 - 2 } & Elipse System Window \\
\cline { 2 - 2 } & Fixed Window System \\
\hline \multirow{4}{*}{4} & Other Window's System \\
\cline { 2 - 2 } & 744 System Door \\
\cline { 2 - 2 } & 8025 System Door \\
\cline { 2 - 2 } & 500 System Window \\
\cline { 2 - 2 } & 3831 System Window \\
\cline { 2 - 2 } & Sliding Window System \\
\hline
\end{tabular}

- Velocity of transporters was set between 0,8 and 1,1 meters per second.
- The simulation entity represents a "production order"; its 116 attributes were programmed according to the statistics provided by the production records of the company for the last three years.

- The simulation model also required designing and programming 119 process stations, 102 resources, 31 production processes, 89 transporters, 1139 routes, 77 variables (23 performance variables and 54 control variables), 515 ARENA modules, 169 ARENA elements, 47 entity and resource pictures, etc. In the same way, the logic of resources downtimes, preventive maintenances, adaptations to new references and the normal flaws of the system were also programmed according to some minor considerations.

\subsection{Construction and Verification of the Model}

The definition of the system and the construction of the conceptual model were carried out with the assistance of the company management and its engineering staff under the guidelines suggested by Bennett [5] and by Harrell, Ghosh and Bowden [8]. A total of 21 spreadsheet files and 14 Power Point, Visio and AutoCAD files were constructed. These files include: plant layout, precedence charts, sub-processes characterization tables, process flow diagrams, material flow and personnel transporting routes, process maps, cause and effect diagrams, correlation matrices, and interstation distance diagrams, among others. Figure 2, for example, shows the process and material flow for the glass cutting department.

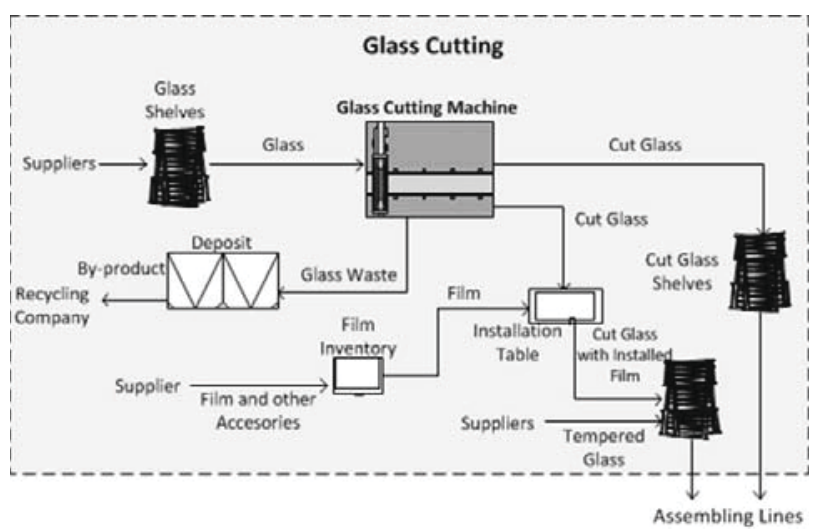

Figure 2. Glass cutting process flow

After the system was defined and the conceptual model was built, the field work was executed in order to extract all the significant quantitative and qualitative information that was required for the programming of the computational model. All the gathered information was statistically analyzed, using the methodologies described by Hines, Montgomery, Corror and Goldsman [9]. The probability distributions were successfully adapted.

More than 17,000 production orders were analyzed. This information helped to prioritize the product references. Moreover, due to the high variance on the processing times, an important number of on-field time samples were taken; 54 on the glass cutting process, 152 on the aluminum cutting process, 581 on the punch pressing and machining, 718 on the assembly process and 
113 on the packing process. Guidance for taking the samples size was provided by Moore (1995) [13].

Using the ARENA 13.9 software and supported by Harrerl, Ghosh and Bowden [8], all structural and operational elements (stations, entities, resources, sequences, operations, arrivals, repairs, etc.) were programmed. 54 unfinished versions of the simulation model were constructed, verified and improved continuously before achieving the final version. The simulation model was programmed in accordance with the process flow sequence. Hence, glass cutting was the first programmed activity and packing was the last one.

The construction and verification stage of the simulation model concluded with the management approval after comparing the behavior of the real system with the computational simulation. Performance variables were finally included into the model before entering the model validation and results analysis phase.

\subsection{Model Validation}

The model validation was carried out by following the 4 main test proposed by Garavito [6]: continuity test, consistency test, absurd conditions test and degeneration test. Table 2 shows a resume of validation test applied to the simulation model.

Table 2. Validation tests applied

\begin{tabular}{|c|c|c|}
\hline Test & Description & Results \\
\hline test & $\begin{array}{c}\text { Applying little changes on input } \\
\text { values must generate } \\
\text { proportional changes on the } \\
\text { outputs }\end{array}$ & $93.99 \%$ \\
\hline $\begin{array}{c}\text { Consistency } \\
\text { test }\end{array}$ & $\begin{array}{c}\text { The variance of a variable within } \\
\text { different simulation runs can not } \\
\text { be higher than } 15 \%\end{array}$ & $8.35 \%$ \\
\hline $\begin{array}{c}\text { Absurd } \\
\text { conditions } \\
\text { test }\end{array}$ & $\begin{array}{c}\text { The simulation model should be } \\
\text { programmed to avoid reproducing } \\
\text { absurd conditions }\end{array}$ & $\begin{array}{c}\text { No absurd conditions were } \\
\text { encountered }\end{array}$ \\
\hline $\begin{array}{c}\text { Degeneration } \\
\text { test }\end{array}$ & $\begin{array}{c}\text { Removing elements from } \\
\text { simulation modeling should result } \\
\text { on a proportional impact }\end{array}$ & $\begin{array}{c}\text { A 30\% reduction of the } \\
\text { assembling resources cuts the } \\
\text { station capacity by } 35.2 \%\end{array}$ \\
\hline
\end{tabular}

Aditional tests presented by Sargent [16] including: face validation, multi-stage validation, operational graphics, crawls and comparison with other models, were also made.

The data structure test was the final validation exam and consisted on statistically comparing the simulation model and the real system. A single factor Anova test was performed for comparing a year production capacity of the simulation model per month and the company's real total orders production for the last year in a monthly manner. This Fisher test suggested a critical factor for $\mathrm{F}$ of 3.0576 and a $\mathrm{F}$ value of 0.8634 , concluding that the simulation model represents a valid copy of the real system.

\subsection{Model Results Analysis}

Once the simulation was concluded, the outgoing results were debugged and compiled, as shown Table 3. This table helped concluding about the system capacity and efficiency.

\subsubsection{System Capacity Analysis}

As noticed in Table 3 , the production plant has a maximum average capacity of $19,590 \mathrm{~m}^{2}$ per month, producing 5,704 products, corresponding to 1,684 manufactured production orders.
Table 2. Simulation model results

\begin{tabular}{|c|c|c|c|}
\hline \multicolumn{4}{|c|}{ PERFORMANCE VARIABLES } \\
\hline \multicolumn{3}{|c|}{ Total Area Capacity } & \multirow{2}{*}{$\begin{array}{r}\text { Average } \\
19.590,2\end{array}$} \\
\hline 1 & Total area produced & {$[\mathrm{m} 2]$} & \\
\hline 2 & Window's area produced & {$[\mathrm{m} 2]$} & $8.102,2$ \\
\hline 3 & Doors area produced & {$[\mathrm{m} 2]$} & $4.733,4$ \\
\hline 4 & Other's area produced & {$[\mathrm{m} 2]$} & $3.411,1$ \\
\hline 5 & Facade's area produced & {$[\mathrm{m} 2]$} & $3.343,5$ \\
\hline \multicolumn{4}{|c|}{ Capacity in number of units } \\
\hline 6 & Production orders & {$[$ Un] } & 1.684 \\
\hline 7 & Units of product & {$[$ Un] } & 5.704 \\
\hline \multicolumn{4}{|c|}{ Process time per production order } \\
\hline 8 & Throughput Time & {$[\text { Days }]^{*}$} & 5,46 \\
\hline 9 & Takt time & [Min] & 10,27 \\
\hline 10 & Value added process time & {$[\mathrm{Hrs}]$} & 13,33 \\
\hline 11 & Portion of value added time & [\%] & $26,1 \%$ \\
\hline \multicolumn{4}{|c|}{ Allocation of resources } \\
\hline 12 & Glass operator leader & [\%] & $71,2 \%$ \\
\hline 13 & Glass operators & [\%] & $72,4 \%$ \\
\hline 14 & Aluminum operators & {$[\%]$} & $99,2 \%$ \\
\hline 15 & Support aluminum operators & [\%] & $99,5 \%$ \\
\hline 16 & Pressing aluminum operators & [\%] & $98,4 \%$ \\
\hline 17 & Copy operators & [\%] & $79,2 \%$ \\
\hline 18 & CNC operators & [\%] & $98,0 \%$ \\
\hline 19 & Assembling line 1 & [\%] & $95,7 \%$ \\
\hline 20 & Assembling line 2 & {$[\%]$} & $93,3 \%$ \\
\hline 21 & Assembling line 3 & [\%] & $98,4 \%$ \\
\hline 22 & Assembling line 4 & [\%] & $92,5 \%$ \\
\hline 23 & Assembling line 5 & [\%] & $92,4 \%$ \\
\hline
\end{tabular}

Furthermore, the capacity analysis was extended to every individual process area. Few changes were made in the simulation model such that the department under analysis was never restricted and could produce the $100 \%$ of the simulated time. The production capacities for the four major processes are presented in Figure 4.

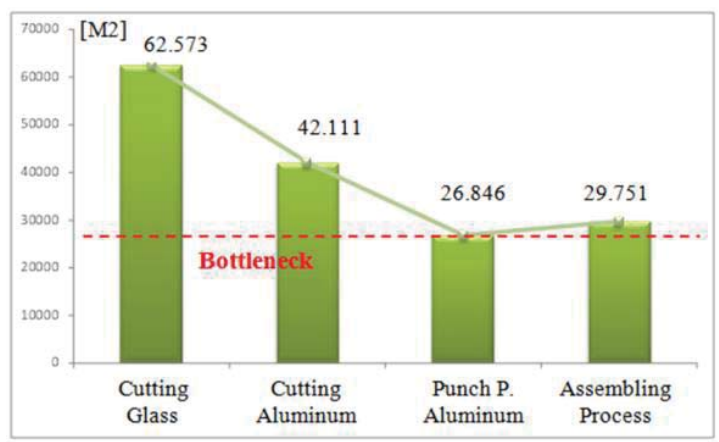

Figure 4. Maximum capacity by process station

In accordance with Figure 4, the punch pressing and machining process represented the "bottleneck" of the system with an approximate capacity of $26,846 \mathrm{~m}^{2}$ per month, followed by the assembly, with a $29,751 \mathrm{~m}^{2} /$ month maximum capacity. In contrast, there was clear evidence that the glass cutting process capacity exceeded the "bottleneck" process in about 133\%. This last fact deduced that, either the Cutting Machine incurred in long idle periods, or the in process inventory is constantly increasing.

Moreover, the punch pressing and machining process were studied in a higher level for determining the main cause of its 
capacity restriction. Its three activities were analyzed separately and an extra variable was programmed for quantifying the average waiting time of in-process products. This measure is shown in Figure 5 .

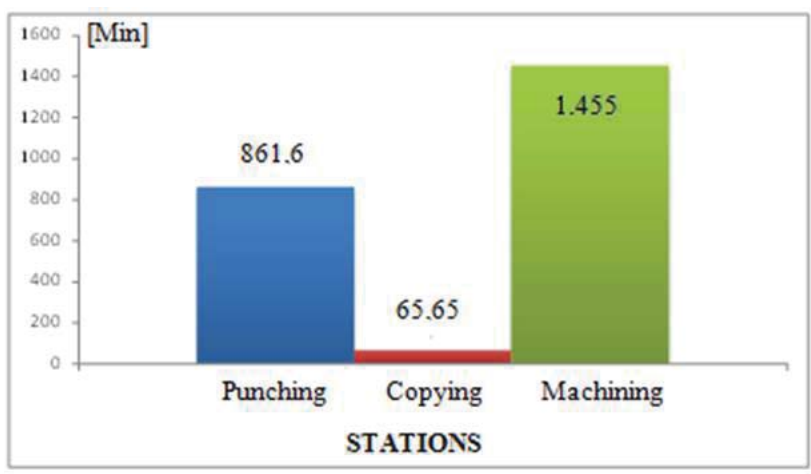

Figure 5. Internal waiting times [min/month] in punch pressing/machining areas

Figure 5 shows that the most critical activity of the manufacturing system was the machining process; therefore, in order to increase the plant's capacity and efficiency, this area had to be the first to be improved.

The initial system analysis through simulation helped also concluding that because of the high difference of capacity levels between the "bottleneck" process $\left(26,846 \mathrm{~m}^{2}\right.$ per month) and the total system capacity, whose value is $19,590 \mathrm{~m}^{2}$ per month, the system required reengineering improvements, essentially in the production logic and interdepartmental coordination.

\subsubsection{Efficiency Evaluation}

The performance variable number 8 , called "throughput time" helped to conclude that the average time to produce an order was equivalent to 5,46 days. This value is the sum of value added time and production time wastes, such as waiting times, transports and in process inventory times. The total amount of time that a production order earns value represented less than $26 \%$ of the 5.46 days.

Another variable called "takt time", measured the time between shipped orders. This average value, of 10.27 minutes, played an important role when comparing further simulation scenarios.

According to the resources occupation, glass cutting showed an occupation of $71.2 \%$, where only $53 \%$ of such time is characterized as value added time. On the other hand, punch pressing and machining process showed an occupation of $98.4 \%$, time constituted by $86 \%$ of value added activities and $14 \%$ of interdepartmental transports.

The assembly department reported an occupation of $94 \%$ of the total productive time, demonstrating a high demand of their work and confirming that this area represent the second less productive of the entire system.

\subsection{Improvement Scenarios}

Having detected the system flaws, there were different scenarios aim to increase the production capacity and the production efficiency of the plant.
Five different scenarios were built and simulated with continuous comparison of the performance variables between the proposed improvements and the initial model.

According to management recommendations, three scenarios were programmed for simulating the production dedicated on only one of the three products (Windows, Doors or Facades). This, with the idea of knowing what if the plant only produced one of the three products and with which, the revenue would be the highest. It resulted that neither would produce higher revenue than the current one.

A fourth scenario was meant to simulate the system coordinating all the stations using "pull" production principles, seeking to: reduce in-process inventory and optimize resource utilization. Some transporting logistics were changed and a minor plant layout was used. This scenario dropped important results that helped on designing the fifth scenario.

The main effort in building the fifth scenario was seeking to achieve the best resource configuration in the punch pressing and machining process. A lot of mathematical iterations were made on a spreadsheet before simulating all the possible optimal scenarios.

After simulating all the proposed models, the scenario with the best performance was chosen. The differences between this model and the initial model follow:

- Both, glass cutting department and punch pressing and machining were synchronized so that the material arrive from these two areas to the assembly department at the same time, reducing substantially the inventory levels.

- A perfect configuration of resources in the punch pressing and machining process was set according to the demand. Some new resources were proposed to be bought for improving the station capacity.

- A new layout in the punch pressing and machining process was proposed. Machines that have a continuous flow were set closed to each other, reducing the transport times and improving the workers occupation levels in value added activities.

- The workers activities were reestablished, so that transports and other waste activities were done by the less paid employees and the most skilled workers dedicated most of their time in value added activities.

- A new double purpose transport units were implemented. Materials were proposed to be stored and transported in adapted "cars" instead of being manually moved. This resulted in waste elimination and enhancing the plant cleaning attributes, among many other benefits.

- A total reengineering on the raw material of aluminum was designed. An inventory model for material consumption and the supply logic of the metal cutting machine was changed so that it was in accordance with the production planning.

As a result, below in table 4 there is a comparison between the initial scenario and the proposed scenario by the analysts.

As noted, the increase in the operational capacity rounds the 18 $\%$ on average and increasing the total amount of product in a $35 \%$. The reduction of idle time improved in $6 \%$.

\section{LIMITATIONS}

The time taking in some production processes required several hours, and in some cases, days to be completed, providing high levels of difficulty in the data collection. Therefore, some minor assumptions were made for minimizing errors. Furthermore, some processing times were divided into micro operations and described by the sum of several probability distributions. For 
example: if an order had operations of engraved and cut, each one was timed for separated regardless the production order belonging. The limitation of this is a discriminated bias that is generated in the data, because the remaining operations time is applied as supplements in percentage to the distribution of times obtained in the analysis.

Table 3. Comparison of current and improved stages

\begin{tabular}{|c|c|c|c|c|c|}
\hline \multicolumn{6}{|c|}{ COMPARISON BETWEEN SCENARIOS } \\
\hline \multicolumn{3}{|c|}{ Total Area Capacity } & Current & Optimal & Delta [\%] \\
\hline 1 & Total area produced & {$[\mathrm{m} 2]$} & $19.590,2$ & $23.073,1$ & $18 \%$ \\
\hline \multicolumn{6}{|c|}{ Capacity in number of units } \\
\hline 7 & Units of product & [Un] & 5.704 & 7.703 & $35 \%$ \\
\hline \multicolumn{6}{|c|}{ Process time per production order } \\
\hline 8 & Throughput Time & [Days] & 5,46 & 3,94 & $-28 \%$ \\
\hline 9 & Takt time & [Min] & 10,27 & 11,02 & $7 \%$ \\
\hline 10 & Value added process time & {$[\mathrm{Hrs}]$} & 13,33 & 13,19 & $-1 \%$ \\
\hline \multicolumn{6}{|c|}{ Allocation of resources } \\
\hline 13 & Glass operators & [\%] & $72,4 \%$ & $59,4 \%$ & $-13 \%$ \\
\hline 14 & Aluminum operators & [\%] & $99,2 \%$ & $98,5 \%$ & $-1 \%$ \\
\hline 15 & Support aluminum operators & [\%] & $99,5 \%$ & $99,5 \%$ & $0 \%$ \\
\hline 16 & Pressing aluminum operators & [\%] & $98,4 \%$ & $94,5 \%$ & $-4 \%$ \\
\hline 17 & Copy operators & [\%] & $79,2 \%$ & $91,6 \%$ & $12 \%$ \\
\hline 18 & CNC operators & [\%] & $98,0 \%$ & $99,9 \%$ & $2 \%$ \\
\hline 19 & Assembling line & [\%] & $94,5 \%$ & $90,1 \%$ & $-4 \%$ \\
\hline
\end{tabular}

\section{RESEARCH CONCLUSIONS}

The simulation model allowed a detailed analysis of the manufacturing plant performance, identifying the most significant weaknesses in the different production with their respective causes.

The global improvement of project was $18 \%$ in capacity which allowed to Ventanar S.A. producing about $4.000 \mathrm{~m}^{2}$ more than its initial production levels. This improvement was achieve with the creation of better synergy between all stations involved and an enhancement of capacity of bottleneck known as punch pressing and machining of aluminum. Also, one of the solutions consents in organizing the production orders, making the process more fluid and concentrating workers on repetitive tasks instead of changing references of product in many opportunities.

Hence, those achievements increase allocation of resources of punch pressing and machining area, improving its performance.

The utility of applying computational simulation models was confirmed. It helped predicting the results of different possible scenarios, achieving conclusions that allowed management to take better investment decisions, maximizing in such way its return.

The initial system analysis helped to develop all the different alternatives for processes improvement. The optimal scenario that achieves the wanted results became a path line in the production floor to follow.

\section{REFERENCES}

[1] Altiok T and Melamed B. (2007), "Simulation Modeling and Analysis with ARENA", Elsevier, London.
[2] Antony J, Hughes M and Kaye M. (1999), "Reducing Manufacturing Process Variability Using Experimental Design Technique: a Case Study”, Integrated Manufacturing System, pp. 162-169.

[3] Azadivar F. (1999), "Simulation Optimization Methodologies", Proceedings of the 1999 Winter Simulation Conference, 93-100.

[4] Banks J. (1998), "Handbook of Simulation: Principles, Methodology, Advances, Applications and Practice", Engineering and Management Press, New Jersey.

[5] Bennett B. (1995), "Simulation Fundamentals", Prentice Hall International, Hemel Hempstead.

[6] Garavito E. (2007), Universidad Industrial de Santander, Industrial Engineering, "Modern Optimization Techniques", Bucaramanga, Colombia.

[7] Gutierrez, J. (1994), "Proyecto de Simulación - Cervecería de Bogotá", Universidad de los Andes, Bogota, Colombia.

[8] Harrel C., Ghosh B. and Bowden R. (2004) "Simulation Using Promodel". United States of America: $2^{\text {nd }}$ Edition. Mc Graw Hill, pp.7, $203-220$.

[9] Hines W., Montgomery D., Goldsman D. and Corror C. (2003), "Probability and Statistics in Engineering", $4^{\text {th }}$ Edition, John Wiley \& Sons, Inc, New Jersey.

[10] Kelton D. (1999) "Designing Simulation Experiments", proceedings of the 1999 Winter Simulation Conference, pp. 3538.

[11] Kelton D., Sadowski R. and Swets N. (2010), "Simulation with ARENA", $5^{\text {th }}$ Edition, McGraw Hill, New York.

[12] Law A. (2007), "Statistical Analysis of Simulation Output Data: The practical State of Art", Proceedings of the 2009 Winter Simulation Conference, pp. 77-83.

[13] Moore D. (1995), "The Basic Practice of Statistics", $2^{\text {nd }}$ Edition. W.H. Freeman and Company, New York, pp. 213-220.

[14] Mosca R, Cassettari L, Revetria R and Gianluca M. (2005), "Simulation as Support for Production Planning in Small and Medium Enterprise: A Case Study", Proceedings of the 2005 Winter Simulation Conference, Kuhl et al (eds), pp. 2443-2448.

[15] Osidach V and Fu M. (2003), "Computer Simulation of a Mobile Examination Centre", Proceedings of the 2003 Winter Simulation Conference, Chick et al (eds), pp. 1868-1875.

[16] Sargent R. (2009), "Verification and Validation of Simulation Models", Proceedings of the 2009 Winter Simulation Conference, pp. 162-176.

[17] Slack N., Chambers S., Johnston R. and Betts A. (2006), "Operations and Process Management, Principles and Practices for Strategic Impact", Prentice Hall-Pearson Education, Harlow, Essex.

[18] Tanigawa Y, Mori H and Watanabe K. (1990), "Computer Simulation of Consistency and Rheology Tests of Fresh Concrete by Viscoplastic Finite Element Method", Proceedings of the Rilem Colloquium, Chapman and Hall, pp. 268-277. 